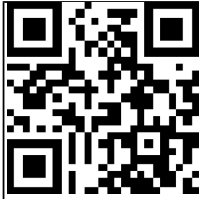

Editor's choice can to access mo free content

- Additional material is published online only. To view please visit the journal online (http://dx.doi.org/10.1136/ bjsports-2014-093976).

${ }^{1}$ Oslo Sports Trauma Research Center, Norwegian School of Sport Sciences, Oslo, Norway ${ }^{2}$ Faculty of Medicine, University of Oslo, Oslo, Norway ${ }^{3}$ Department of Cardiology, Oslo University Hospital, Ullevål, Oslo, Norway

\section{Correspondence to} Dr H M Berge, Oslo Sports Trauma Research Center, Norwegian School of Sport Sciences, Sognsveien 220, PO Box 4014 Ullevål stadion, Oslo N0-0806 Norway;

hilde.moseby.berge@nih.no

$\mathrm{HMB}$ and $\mathrm{CBI}$ contributed equally.

Accepted 23 December 2014 Published Online First 28 January 2015

\title{
Blood pressure and hypertension in athletes: a systematic review
}

\author{
H M Berge, ${ }^{1}$ C B Isern, ${ }^{2}$ E Berge $^{3}$
}

\section{ABSTRACT}

Objective Hypertension is reported to be the most prevalent risk factor for cardiovascular disease in elite athletes. We aimed to review blood pressure (BP) and prevalence of hypertension in different elite athletes, and study whether there was an association between high $\mathrm{BP}$ and left ventricular hypertrophy (LVH).

Methods A systematic review of studies reporting BP in athletes using search strategies developed for PubMed and EMBASE, including only studies with $\geq 100$ participants. We collected data on $\mathrm{BP}$, prevalence of hypertension, LVH and methods of BP measurement. Results Of 3723 records identified, 51 met the inclusion criteria. These included men and women $(n=138390)$, aged mostly between 18 and 40 years, from varied sports disciplines. Mean systolic BP varied from $109 \pm 11$ to $138 \pm 7 \mathrm{~mm} \mathrm{Hg}$ and mean diastolic BP from $57 \pm 12$ to $92 \pm 10 \mathrm{~mm} \mathrm{Hg}$. Strength-trained athletes had higher BP than endurance-trained athletes (131.3 $\pm 5.3 / 77.3 \pm 1.4$ vs $118.6 \pm 2.8 / 71.8 \pm 1.2 \mathrm{~mm} \mathrm{Hg}$, $\mathrm{p}<0.05)$, and there was a trend towards a higher BP in athletes training $\geq 10 \mathrm{~h}$ compared with others (121.8 $\pm 3.8 / 73.8 \pm 2.5$ vs $117.6 \pm 3.3 / 66.8 \pm 6.9, p=0.058)$, but overall there was no significant difference in BP between athletes and controls. The prevalence of hypertension varied from $0 \%$ to $83 \%$. Some studies showed an association between high BP and LVH. Measurement methods were poorly standardised.

Conclusions BP and prevalence of hypertension in athletes varied considerably partly because of variations in methodology, but type and intensity of training may contribute towards higher BP. High BP may be associated with LVH.

\section{INTRODUCTION}

In Western countries, the prevalence of hypertension has been reported as $14.4 \%$ and $21.2 \%$ in men aged 20-29 and 30-39 years, respectively, and as $6.2 \%$ and $9.9 \%$ in women in the same age group. ${ }^{1}$ High blood pressure (BP) at a young age predicts cardiovascular mortality and morbidity decades later. ${ }^{23}$

High BP is the most common abnormal finding during preparticipation cardiac screening of athletes. ${ }^{4-8}$ The prognostic significance of high BP in athletes is unknown, but still athletes with $\mathrm{BP}<160$ / $100 \mathrm{~mm} \mathrm{Hg}$ are given the license to continue with sport participation if they have no signs of end organ damage, such as pathological left ventricular hypertrophy. ${ }^{9}$ Increased left ventricular mass is considered as subclinical organ damage in people with hypertension. ${ }^{10} 11$ As several studies have demonstrated increased left ventricular mass and increased left atrium size in athletes, ${ }^{12}$ it is possible that high BP

\section{What this study adds?}

- There was no evidence that blood pressure (BP) was lower in athletes than in controls. A number of studies showed a higher BP in athletes. The prevalence of hypertension in athletes could not be determined reliably because of different definitions of hypertension and poorly standardised methods of BP measurement.

- A positive linear association between high BP and left ventricular hypertrophy was observed in athletes, but confounding factors may have played a role.

- Future studies should be designed to determine more precisely the prevalence, determinants and prognostic significance of hypertension in athletes.

may be a contributing factor ${ }^{13} 14$ that may also link to the increased risk of atrial fibrillation in endurance athletes. ${ }^{15-17}$ Hence, there is increasing interest in BP in athletes. ${ }^{14} 1819$ BP measurement during preparticipation screening of athletes should be performed according to 'best clinical care', ${ }^{20}$ as outlined in the European Society of Cardiology's guidelines, with hypertension defined as systolic BP (SBP) $\geq 140 \mathrm{~mm} \mathrm{Hg}$ and/or diastolic BP (DBP) $\geq 90 \mathrm{~mm} \mathrm{Hg}$ after repeated measurements. ${ }^{21}$ We aimed to review BP and prevalence of hypertension in different athletes, and study the association between increasing $\mathrm{BP}$ and left ventricular hypertrophy.

\section{METHODS}

\section{Literature search}

We performed a systematic review of studies reporting BP in athletes by using a comprehensive search strategy developed for PubMed and EMBASE (see online supplementary material). The medical subject headings and text words were: 'Athlete', 'Sport and Professional', 'Exercise Test' and 'Sudden Death', combined with 'Blood Pressure' or 'Hypertension'. The electronic search was restricted to studies published before 6 April 2014. In addition, we manually searched reference lists of reviews and original study articles, and our own archive.

\section{Inclusion and exclusion criteria}

We searched for studies of athletes that reported BP or prevalence of hypertension, using the studies' own definitions of hypertension. We included studies of $\geq 100$ athletes, with mean or median age between 18 and 40 years, of any epidemiological design (with or without follow-up, and with or 
without controls), and reported in English language. We excluded studies that were only presented as conference abstracts. If there were more than one publication from the same group, we used the record with most participants, or the newest, if the number of participants were the same. When in doubt, we contacted the corresponding authors.

\section{Outcome variables}

The primary outcome variable was BP or prevalence of hypertension in different categories of athletes (defined by gender, ethnicity, sports discipline or level of athletic activity). Secondary outcome variables were (1) method for measurement of BP and (2) association between BP and left ventricular hypertrophy (determined by left ventricular mass or relative wall thickness on echocardiography or by voltage criteria on ECG).

\section{Extraction of data}

All data were extracted by one reviewer (CBI) and checked by another reviewer (HMB), using a standardised data extraction sheet.

\section{Statistical analysis}

Differences between subgroups of athletes were analysed using $\mathrm{t}$ tests for continuous variables. Data are presented as mean with SD. A p $<0.05$ was considered statistically significant and all tests were two-tailed. The statistical analyses were conducted using SPSS (PASW Statistics 21; IBM Corporation 2013, Armonk, New York, USA).

\section{RESULTS}

\section{Study selection}

The searches retrieved a total of 4433 records (figure 1). After addition of studies from other sources and removal of duplicates, 3723 records remained. Screening of titles and abstracts excluded 2896 and 361, respectively. Another 404 studies did not meet the inclusion criteria, 9 were duplicate reports, and 2 studies were not available. The remaining 51 studies were included in the review.

\section{Study characteristics}

Table 1 shows characteristics of the 51 studies, including a total of 138390 athletes, with a median number of 434 athletes (range $100^{22}-42386$ athletes ${ }^{23}$ ). Sixteen studies included non-athletes as controls and the median number of controls was 176 (range 26-9997). The mean or median age of the athletes in all studies was between 18 and 40 years, and about half of the studies had participants within this range only; however, several studies included participants with an age outside this range. Twenty studies included males only and across the 31 studies of both genders, $72.5 \%$ were males.

Most studies (28) included athletes from different sports disciplines, but 16 included athletes from only one discipline, eg, soccer (4), ${ }^{13}$ 24-26 American football (3), ${ }^{27-29}$ triathlon (2) ${ }^{30} 31$ and long distance running (2); ${ }^{16} 32$ other studies classified sports disciplines as either endurance sports, strength sports or a mixture of the two (table 1). The athletes' level of competition was described in 50 studies and ranged from participation in amateur sport to the Olympic Games. Hours of training per week or previous years of vigorous training were given in 24 studies, and ranged from 4 to $28 \mathrm{~h}$ a week and from 2 to 30 years, respectively.

\section{$\mathrm{BP}$ in athletes}

Table 2 shows BP and prevalence of hypertension in the same studies. Among the 34 studies that reported BP, two-thirds had $\mathrm{BP}$ in the prehypertensive range (SBP $\geq 120-139$ and/or DBP $\geq 80-89 \mathrm{~mm} \mathrm{Hg}) .{ }^{33}$ Mean SBP varied from $109 \pm 11 \mathrm{~mm} \mathrm{Hg}$ (intercollegiate female college athletes (mean age 20 years)) ${ }^{34}$ to

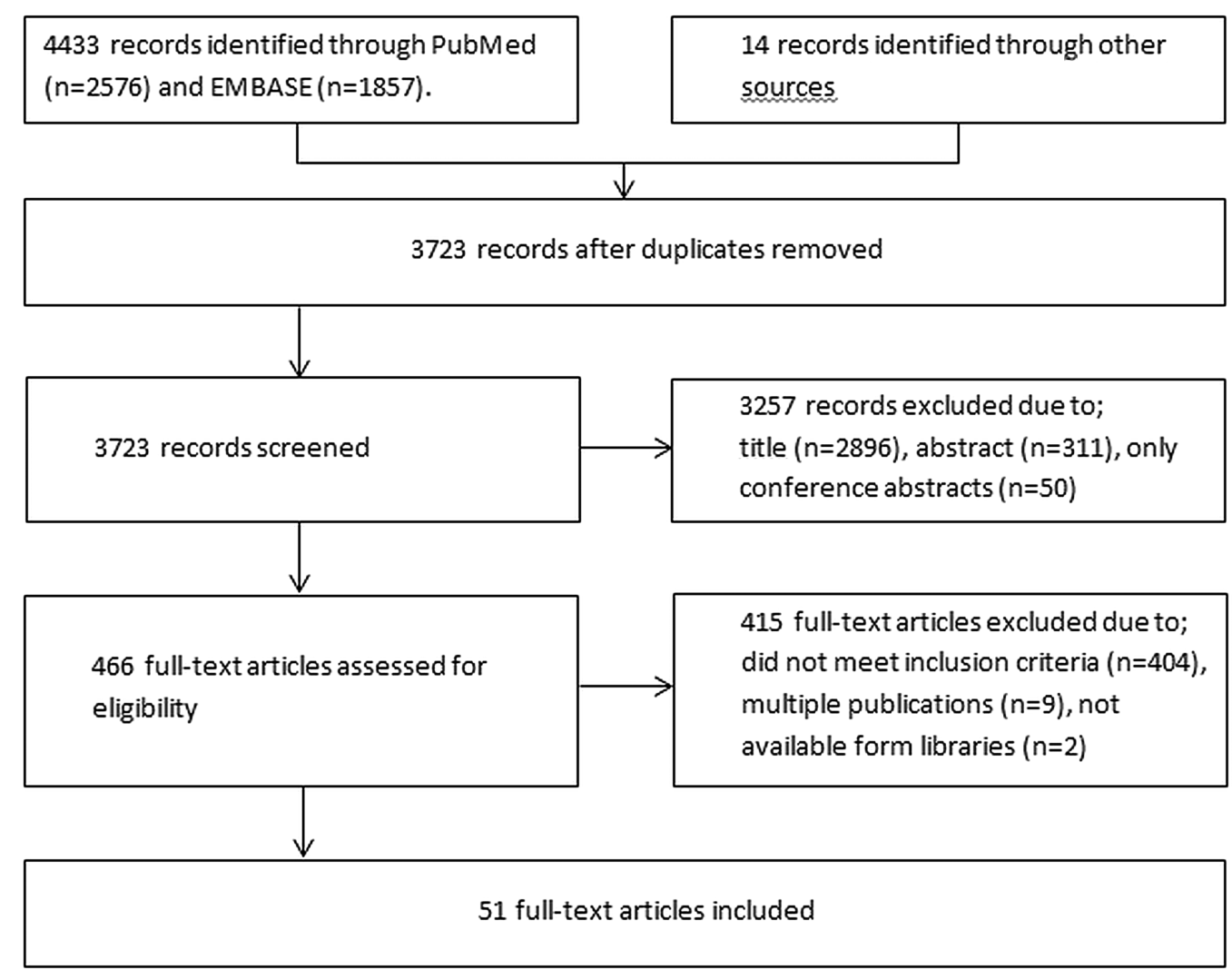

Figure 1 Flow chart illustrating search strategy. 


\begin{tabular}{|c|c|c|c|c|c|c|c|}
\hline $\begin{array}{l}\text { First author, year of } \\
\text { publication }\end{array}$ & $\begin{array}{l}\text { Number of athletes } \\
\text { (males \%) }\end{array}$ & Age (mean $\pm S D$ (range, in years) & Sports disciplines & Level & $\begin{array}{l}\text { Hours of training per } \\
\text { week }\end{array}$ & $\begin{array}{l}\text { Previous years of } \\
\text { training }\end{array}$ & $\begin{array}{l}\text { Number of } \\
\text { controls }\end{array}$ \\
\hline Helzberg, $2010^{37}$ & 224 & $24(18-36)$ & $\begin{array}{l}\text { Football and } \\
\text { baseball }\end{array}$ & Professional & & & \\
\hline van Buuren, $2013^{63}$ & $291(100)$ & $25.3 \pm 4.4(18-39)$ & Handball & Top level athletes & & & \\
\hline Lively, $1999^{5}$ & $596(68.0)$ & $18.9(17-25)$ & Mixed & Intercollegiate & & & \\
\hline Sofi, $2008^{64}$ & $30065(78.4)$ & $30.7 \pm 14(5-92)$ & Mixed & $?$ & $4-6$ & & \\
\hline Pelliccia, $2010^{40}$ & $114(78)$ & $22 \pm 4$ & Endurance & Olympic & 28 & 16 & \\
\hline Munoz, $2009^{65}$ & $135(41)$ & $20(17-25)$ & Mixed & $\begin{array}{l}\text { Intercollegiate, NCAA } \\
\text { Division II }\end{array}$ & & & \\
\hline De Matos, $2011^{6}$ & $623(85)$ & NA (13-77) & Mixed & Professional and amateur & & & \\
\hline Berge, $2013^{13}$ & $595(100)$ & $25.1 \pm 4.5(18-40)$ & Soccer & Professional soccer & & & 47 \\
\hline Weiner, $2013^{14}$ & $183(100)$ & $19 \pm 1(\geq 18)$ & Football and rowing & College & 8 & & \\
\hline Tucker, $2009^{29}$ & $504(100)$ & 26.7 & Football & NFL & & & 1959 \\
\hline Guo, $2013^{34}$ & $261(50.2)$ & 21 & Strength & Professional & & $5-7$ & \\
\hline Karpinos, $2013^{18}$ & $636(100)$ & $18.7 \pm 0.8$ & Mixed & Mixed & & & \\
\hline Lewis, $1989^{66}$ & $265(83)$ & $19(18-28)$ & Mixed & Intercollegiate & & & \\
\hline Corrado, $2006^{23}$ & 42386 & $12-35$ & Mixed & Competitive & & & \\
\hline Thunenkotter, $2010^{28}$ & $582(100)$ & $26.8 \pm 4$ & Football & FIFA World Cup 2006 & & & \\
\hline Wilson, $2012^{59}$ & $1220(100)$ & $22.6 \pm 6(12-35)$ & Mixed & National & $\geq 6$ & & \\
\hline Gati, $2013^{43}$ & $2533(72.2)$ & $21.8 \pm 5.7$ & Mixed & Regional to national & 19 & & 9997 \\
\hline Zaidi, $2013^{39}$ & $627(69.9)$ & $21.5 \pm 5.0(14-35)$ & Mixed & Regional to international & 20 & & \\
\hline Riding, $2013^{49}$ & 1175 & $\begin{array}{l}\text { Arabic: } 22.7 \pm 5.9, \text { BA: } 24.6 \pm 4.7, \text { WA: } 24.4 \pm 5.4 \text {. } \\
(13-40)\end{array}$ & Mixed & High-level & $\geq 6$ & & 201 \\
\hline Di Luigi, $2004^{67}$ & $32652(80)$ & $22.3 \pm 12.5(5-84)$ & Mixed & Competitive & $<10$ & & \\
\hline Magalski, $2011^{68}$ & $964(48)$ & $18-21$ & Mixed & Mixed & & & \\
\hline Papadakis, $2011^{41}$ & $2745(100)$ & NA (14-35) & Mixed & Regional to international & $13-15$ & & 119 \\
\hline Schmied, $2013^{25}$ & $210(100)$ & $18.6(18-22)$ & Soccer & $\begin{array}{l}\text { High-level, competitive } \\
\text { soccer }\end{array}$ & & & \\
\hline Maron, $1987^{69}$ & $501(71.3)$ & $19.3(17-30)$ & Mixed & Intercollegiate & & & \\
\hline Rontoyannis, $1998^{27}$ & $188(100)$ & $36.4 \pm 4.5$ & Football referees & Greece football division A-D & & & \\
\hline Urhausen, $1996^{70}$ & $135(47.4)$ & of $20.6 \pm 4.1$, ᄋ $21.5 \pm 3.4$ & Rowing & National and regional level & & & \\
\hline Pelliccia, $2000^{71}$ & $1005(74)$ & $23(9-55)$ & Mixed & National team & & $2-30$ & \\
\hline Maskhulia, $2006^{24}$ & $221(100)$ & $22.8 \pm 0.3(18-35)$ & Soccer & Highly trained & & $5-26$ & \\
\hline Caselli, $2011^{42}$ & $434(78)$ & $26 \pm 5(15-45)$ & Mixed & Olympic & & $\geq 3$ & 98 \\
\hline Noseworthy, $2011^{36}$ & $879(62)$ & $18.4 \pm 0.8$ & Mixed & Intercollegiate level & 7 & & \\
\hline Varga-Pinter, $2011^{45}$ & $3697(61)$ & $23.7 \pm 4.6(19-40)$ & Mixed & Low to top level & $10-13$ & & \\
\hline Pougnet, $2012^{72}$ & $200(86)$ & $38(19-57)$ & Diving & Professional & & & \\
\hline Schmied, $2012^{73}$ & 1047 (49) & $22 \pm 5.87(13-64)$ & Mixed & Competitive & & & \\
\hline Zaidi, $2013^{38}$ & $675(80.6)$ & BA: $21.8 \pm 5.4, W A: 21.7 \pm 4.6$ & Mixed & Regional to international & $17-20$ & & \\
\hline Berry, $1949^{46}$ & $201(100)$ & NA (16-47) & Mixed & Olympic & & & \\
\hline Andersen, $1956^{48}$ & $326(100)$ & $20-29$ years: $208,30-39$ years: 118 & Mixed & Mixed and national level & & & 526 \\
\hline Siegel, $1992^{44}$ & $1061(100)$ & $\begin{array}{l}<20 \text { years; } 105,20-29 \text { years; } 807,30-39 \text { years; } \\
99,>40 \text { years; } 42\end{array}$ & Baseball & $\begin{array}{l}\text { Professional and minor } \\
\text { league }\end{array}$ & & & \\
\hline
\end{tabular}


Table 1 Continued

\begin{tabular}{|c|c|c|c|c|c|c|c|}
\hline $\begin{array}{l}\text { First author, year of } \\
\text { publication }\end{array}$ & $\begin{array}{l}\text { Number of athletes } \\
\text { (males \%) }\end{array}$ & Age (mean \pm SD (range, in years) & Sports disciplines & Level & $\begin{array}{l}\text { Hours of training per } \\
\text { week }\end{array}$ & $\begin{array}{l}\text { Previous years of } \\
\text { training }\end{array}$ & $\begin{array}{l}\text { Number of } \\
\text { controls }\end{array}$ \\
\hline Douglas, $1997^{30}$ & $140(73)$ & $30 \pm 1(18-39)$ & Triathlon & Ironman, Hawaii & 21 & & \\
\hline$D^{\prime}$ Andrea, $2002^{35}$ & $263(60.5)$ & E: $28.1 \pm 4.2$, S: $27.2 \pm 5.4$ & $\begin{array}{l}\text { Endurance and } \\
\text { strength }\end{array}$ & Top competitive & $15-20$ & 4 & \\
\hline Abergel, $2004^{74}$ & $286(100)$ & $28.4 \pm 3.2$ & Cycling & Professional & & & 52 \\
\hline Sharwood, $2004^{31}$ & 148 & & Triathlon & Ironman, South Africa & & & \\
\hline Maldonado, $2006^{26}$ & $212(100)$ & $20 \pm 5.7$ & Soccer & Competitive & 15 & $2-25$ & 211 \\
\hline Babaee Bigi, $2007^{22}$ & $100(100)$ & $22.1 \pm 3.6$ & Strength & Professional & & & 128 \\
\hline Basavarajaiah, $2008^{75}$ & $600(100)$ & BA: $20.5 \pm 5.8$, WA: $20.2 \pm 4.9 .(14-35)$ & Mixed & National level & 14 & & 150 \\
\hline Molina, $2008^{16}$ & $183(100)$ & $39 \pm 9$ & $\begin{array}{l}\text { Long distance } \\
\text { running }\end{array}$ & Marathon runners & & & 305 \\
\hline Miranda-Vilela, $2009^{32}$ & $125(60.8)$ & $(15-67)$ & $\begin{array}{l}\text { Long distance } \\
\text { running }\end{array}$ & Trained athletes & & & \\
\hline D'Andrea, $2012^{47}$ & $410(70.7)$ & E: $28.7 \pm 10.7$, S: $29.2 \pm 11.2 .(18-40)$ & $\begin{array}{l}\text { Endurance and } \\
\text { strength }\end{array}$ & Elite & $15-20$ & 4 & 240 \\
\hline Pagourelias, $2013^{76}$ & $108(100)$ & E: $31.2 \pm 10.4$, S: $27.4 \pm 5.7$ & $\begin{array}{l}\text { Endurance and } \\
\text { strength }\end{array}$ & $\begin{array}{l}\text { Moderately and highly } \\
\text { trained }\end{array}$ & $15-17$ & $10-11$ & 26 \\
\hline Vitarelli, $2013^{77}$ & 105 & E: $28.7 \pm 10.7$, S: $30.3 \pm 9.4$, Mix: $29.4 \pm 9.8$ & $\begin{array}{l}\text { Endurance and } \\
\text { strength }\end{array}$ & Competitive & 15 & 6 & 35 \\
\hline Malhotra, $2011^{53}$ & $1473(49.7)$ & đ: $19 \pm 2$, ᄋ: $19 \pm 2$ & Mixed & NCAA Division I & & & \\
\hline Chandra, $2014^{78}$ & $4081(80.5)$ & $19.5 \pm 5.2(14-35)$ & Mixed & Regional to international & 15 & & 7764 \\
\hline
\end{tabular}

\%, women; D, men; BA, black athletes; E, endurance; NCAA, National Collegiate Athletic Association; S, strength; WA, white athletes. 
Table 2 Blood pressure and prevalence of hypertension

\begin{tabular}{|c|c|c|c|c|c|c|c|}
\hline \multirow[b]{2}{*}{$\begin{array}{l}\text { First author, year of } \\
\text { publication }\end{array}$} & \multirow[b]{2}{*}{$\begin{array}{l}\text { Definition of } \\
\text { hypertension }\end{array}$} & \multicolumn{4}{|c|}{ Athletes } & \multicolumn{2}{|l|}{ Controls } \\
\hline & & N & $\begin{array}{l}\text { Percentage with } \\
\text { hypertension }\end{array}$ & $\begin{array}{l}\text { SBP }(\text { mean } \pm S D \text {, } \\
\mathrm{mm} \mathrm{Hg})\end{array}$ & $\begin{array}{l}\text { DBP (mean } \pm S D \text {, } \\
\mathrm{mm} \mathrm{Hg})\end{array}$ & $\begin{array}{l}\text { SBP } \\
\text { (mean, mm Hg) }\end{array}$ & $\begin{array}{l}\text { DBP } \\
\text { (mean, mm Hg) }\end{array}$ \\
\hline Helzberg, $2010^{37}$ & $\geq 130 / 85$ & 101 & 45.1 & & & & \\
\hline van Buuren, $2013^{63}$ & $>135 / 80$ & 3 & 1.0 & $125.7 \pm 17.8$ & $77.3 \pm 10.6$ & & \\
\hline Lively, $1999^{5}$ & $\geq 140 / 90$ & 22 & 3.7 & & & & \\
\hline Sofi, $2008^{64}$ & $\geq 140 / 90$ & 37 & 0.1 & & & & \\
\hline Pelliccia, $2010^{40}$ & $\geq 140 / 90$ & 0 & 0.0 & & & & \\
\hline Munoz, $2009^{65}$ & $\begin{array}{l}\text { SBP } \geq 140 \text { and/or DBP } \\
\geq 90\end{array}$ & 3 & 2.2 & đ $117 \pm 12$, ᄋ $109 \pm 11$ & ot $73 \pm 10$, ㅇ $72 \pm 8$ & & \\
\hline De Matos, $2011^{6}$ & $\begin{array}{l}\text { SBP } \geq 140 \text { and/or DBP } \\
\geq 90\end{array}$ & 49 & 7.9 & & & & \\
\hline Berge, $2013^{13}$ & $\begin{array}{l}\text { SBP } \geq 140 \text { and/or DBP } \\
\geq 90\end{array}$ & 39 & 7.0 & $122 \pm 11$ & $69 \pm 8$ & 123.8 & 70.8 \\
\hline Weiner, $2013^{14}$ & $\begin{array}{l}\mathrm{SBP} \geq 140 \text { and/or DBP } \\
\geq 90\end{array}$ & 1 & 0.5 & $\begin{array}{l}\text { Football: } 116 \pm 8, \\
\text { Rowing: } 114 \pm 9\end{array}$ & $\begin{array}{l}\text { Football: } 64 \pm 8, \\
\text { Rowing: } 60 \pm 9\end{array}$ & & \\
\hline Tucker, $2009^{29}$ & $\begin{array}{l}S B P \geq 140 \text { and/or DBP } \\
\geq 90 \text {, or anti-HT } \\
\text { medication }\end{array}$ & 67 & 13.8 & 127 & 75 & 112.0 & 72.0 \\
\hline Guo, $2013^{34}$ & $\begin{array}{l}\mathrm{SBP} \geq 140 \text { and/or } \mathrm{DBP} \\
\geq 90, \text { or anti-HT } \\
\text { medication }\end{array}$ & 56 & 41.9 & $\begin{array}{l}\text { LBW: }{ }^{\star} 121.2 \pm 9.0, \text { } \\
110.8 \pm 10.1 \text { UBW: }{ }^{\star} \\
137.4 \pm 13.8, \text { o } 124.6 \\
\pm 15.2\end{array}$ & $\begin{array}{l}\text { LBW: o } 81.5 \pm 9.0, \text { } \\
73.6 \pm 8.2 \text { UBW: o } 92.2 \\
\pm 9.6, \text { o } 83.8 \pm 12.0\end{array}$ & & \\
\hline Karpinos, $2013^{18}$ & $\begin{array}{l}\mathrm{SBP} \geq 140 \text { and/or DBP } \\
\geq 90, \text { or anti-HT } \\
\text { medication, or } \\
\text { self-reported HT }\end{array}$ & 84 & 13.2 & $\begin{array}{l}\text { Football: } 126.4 \pm 11.0 \\
\text { Non-football: } 122.5 \pm 9.8\end{array}$ & $\begin{array}{l}\text { Football: } 75.3 \pm 9.9 \\
\text { Non-football: } 72.3 \pm 9.0\end{array}$ & & \\
\hline Lewis, $1989^{66}$ & $>140 / 90$ & 4 & 1.5 & & & & \\
\hline Corrado, $2006^{23}$ & $>140 / 90$ & 205 & 0.5 & & & & \\
\hline Thunenkotter, $2010^{28}$ & $>140 / 90$ & 12 & 2.1 & $119 \pm 11(\leq 190)$ & $73 \pm 9(\leq 105)$ & & \\
\hline Wilson, $2012^{59}$ & $>140 / 90$ & 10 & 0.8 & & & & \\
\hline Gati, $2013^{43}$ & $>140 / 90$ & 12 & 0.5 & $120 \pm 12.6$ & & 115.0 & \\
\hline Zaidi, $2013^{39}$ & $>140 / 90$ & 0 & 0.0 & & & & \\
\hline Riding, $2013^{49}$ & $\mathrm{SBP}>140$ & 17 & 1.4 & $\begin{array}{l}\text { Arabic: } 120 \pm 13, \text { BA: } \\
125.2 \pm 10.9, \text { WA: } 126.4 \\
\pm 11.8\end{array}$ & $\begin{array}{l}\text { Arabic: } 71 \pm 9, \text { BA: } 73.6 \\
\pm 8.4, \text { WA: } 74.5 \pm 10.1\end{array}$ & 118.1 & 72.1 \\
\hline Di Luigi, $2004^{67}$ & $\begin{array}{l}\mathrm{SBP}>140 \text { and/or DBP } \\
>90\end{array}$ & 587 & 1.8 & $\begin{array}{l}\text { of } 119.8 \pm 12.5 \text {, ᄋ } 115.4 \\
\pm 12.1\end{array}$ & $\begin{array}{l}\text { of } 72.8 \pm 9.3, \text { o } 70.2 \\
\pm 9.0\end{array}$ & & \\
\hline Magalski, $2011^{68}$ & $\begin{array}{l}\mathrm{SBP}>140 \text { and/ or DBP } \\
>90\end{array}$ & 26 & 2.7 & & & & \\
\hline Papadakis, $2011^{41}$ & $\begin{array}{l}\mathrm{SBP}>140 \text { and/or DBP } \\
>90\end{array}$ & 22 & 0.8 & $\begin{array}{l}\text { BA: } 116.5 \pm 13.1, W A: \\
111.8 \pm 11.0\end{array}$ & & 121.7 & \\
\hline Schmied, $2013^{25}$ & $\begin{array}{l}\text { SBP }>140 \text { and/or DBP } \\
>90\end{array}$ & 12 & 5.7 & $125 \pm 4$ & $72 \pm 2$ & & \\
\hline Maron, $1987^{69}$ & $>145 / 90$ & 1 & 0.2 & & & & \\
\hline Rontoyannis, $1998^{27}$ & $\geq 160 / 95$ & 18 & 9.5 & & & & \\
\hline Urhausen, $1996^{70}$ & & 0 & 0.0 & & & & \\
\hline Pelliccia, $2000^{71}$ & & 3 & 0.3 & & & & \\
\hline Maskhulia, $2006^{24}$ & & 2 & 0.9 & & & & \\
\hline Caselli, $2011^{42}$ & & 1 & 0.2 & $117 \pm 10$ & $76 \pm 6$ & 119.0 & 75.0 \\
\hline Noseworthy, $2011^{36}$ & & 3 & 0.3 & $116.6 \pm 8.6$ & $56.9 \pm 11.5$ & & \\
\hline Varga-Pinter, $2011^{45}$ & & 0 & 0.0 & o $126 \pm 13$, 우 $116 \pm 13$ & ơ $80 \pm 10$, ㅇ $75 \pm 13$ & & \\
\hline Pougnet, $2012^{72}$ & & 32 & 16.0 & 123.4 & 77.5 & & \\
\hline Schmied, $2012^{73}$ & & 2 & 0.2 & & & & \\
\hline Zaidi, $2013^{38}$ & $>120 / 80$ & 0 & 0.0 & & & & \\
\hline Berry, $1949^{46}$ & & & & $119.1 \pm 13.1$ & $77.4 \pm 8.1$ & & \\
\hline Andersen, $1956^{48}$ & & & & $\begin{array}{l}20-29 \text { years: } 132.5 \\
\pm 15.6,30-39 \text { years: } \\
133.8 \pm 14.1\end{array}$ & $\begin{array}{l}\text { 20-29 years: } 80.6 \\
\pm 10.9,30-39 \text { years: } \\
83.0 \pm 3.4\end{array}$ & 135.6 & 85.4 \\
\hline Siegel, $1992^{44}$ & & & & 117.1 & 71.8 & & \\
\hline Douglas, $1997^{30}$ & & & & $122 \pm 1$ & $74 \pm 1$ & & \\
\hline$D^{\prime}$ Andrea, $2002^{35}$ & & & & $\begin{array}{l}\text { E: } 116.8 \pm 9.1, S: 137.9 \\
\pm 7.1\end{array}$ & $\begin{array}{l}\text { E: } 72.7 \pm 4.9, \text { S: } 78.9 \\
\pm 4.0\end{array}$ & & \\
\hline Abergel, $2004^{74}$ & & & & $120 \pm 9$ & $68 \pm 9$ & 126.0 & 77.0 \\
\hline
\end{tabular}


Table 2 Continued

\begin{tabular}{|c|c|c|c|c|c|c|c|}
\hline \multirow[b]{2}{*}{$\begin{array}{l}\text { First author, year of } \\
\text { publication }\end{array}$} & \multirow[b]{2}{*}{$\begin{array}{l}\text { Definition of } \\
\text { hypertension }\end{array}$} & \multicolumn{4}{|c|}{ Athletes } & \multicolumn{2}{|l|}{ Controls } \\
\hline & & $\mathbf{N}$ & $\begin{array}{l}\text { Percentage with } \\
\text { hypertension }\end{array}$ & $\begin{array}{l}\text { SBP (mean } \pm S D \text {, } \\
\mathrm{mm} \mathrm{Hg)}\end{array}$ & $\begin{array}{l}\text { DBP (mean } \pm S D \text {, } \\
\text { mm Hg) }\end{array}$ & $\begin{array}{l}\text { SBP } \\
\text { (mean, mm Hg) }\end{array}$ & $\begin{array}{l}\text { DBP } \\
\text { (mean, mm Hg) }\end{array}$ \\
\hline Sharwood, $2004^{31}$ & & & & $133 \pm 12$ & $78 \pm 9$ & & \\
\hline Maldonado, $2006^{26}$ & & & & $127 \pm 7.2$ & $70 \pm 9.0$ & 130.0 & 73.0 \\
\hline Babaee Bigi, $2007^{22}$ & & & & $137.5 \pm 5.7$ & $86.2 \pm 6.5$ & & \\
\hline Basavarajaiah, $2008^{75}$ & & & & BA: $118 \pm 7$, WA: $115 \pm 6$ & & 119.0 & \\
\hline Molina, $2008^{16}$ & & & & $125 \pm 15$ & $76 \pm 9$ & 133.0 & 80.0 \\
\hline Miranda-Vilela, $2009^{32}$ & & & & $\begin{array}{l}\text { đ } 117.1 \pm 0.9 \text {, o } 112.0 \\
\pm 1.0\end{array}$ & $\begin{array}{l}\text { o } 74.7 \pm 1.0, \text { ㅇ } 69.3 \\
\pm 1.1\end{array}$ & & \\
\hline D'Andrea, $2012^{47}$ & & & & $\begin{array}{l}\text { E: } 115.8 \pm 6.1, \text { S: } 132.9 \\
\pm 8.1\end{array}$ & $\begin{array}{l}\text { E: } 72.7 \pm 4.9, \text { S: } 75.9 \\
\pm 4.0\end{array}$ & 120.3 & 74.6 \\
\hline Pagourelias, $2013^{76}$ & & & & $\mathrm{E}: 120 \pm 7, \mathrm{~S}: 125.4 \pm 8$ & $\mathrm{E}: 70.3 \pm 8.5, \mathrm{~S}: 78 \pm 3$ & 120.6 & 75.0 \\
\hline Vitarelli, $2013^{77}$ & & & & $\begin{array}{l}\text { E: } 121.9 \pm 6.8, \text { S: } 129.1 \\
\pm 7.4, \text { mix: } 123.2 \pm 7.1\end{array}$ & $\begin{array}{l}\text { E: } 71.3 \pm 5.4, \text { S: } 76.3 \\
\pm 4.8, \text { mix: } 70.9 \pm 4.6\end{array}$ & 120.6 & 75.2 \\
\hline Malhotra, $2011^{53}$ & & & & o $126 \pm 12$, ㅇ $115 \pm 11$ & o $75 \pm 9$, \& $73 \pm 22$ & & \\
\hline Chandra, $2014^{78}$ & & & & $114.5 \pm 13.1$ & $69.3 \pm 21.8$ & 120.6 & 69.5 \\
\hline
\end{tabular}

$137.9 \pm 7.1 \mathrm{~mm} \mathrm{Hg}$ (Italian male strength sports athletes (mean age 27.2 years)). ${ }^{35}$ Mean DBP ranged from $56.9 \pm 11.5 \mathrm{~mm} \mathrm{Hg}$ (young college level athletes in the USA (mean age 18.4 years) $)^{36}$ to $92.2 \pm 9.6 \mathrm{~mm} \mathrm{Hg}$ (male Chinese strength sports athletes with mean body weight $130 \mathrm{~kg}$ (mean age 21.7 years)). ${ }^{34}$ No studies reported ambulatory BP measurements.

Among the 16 studies that included non-athletes as controls, BP was lower in athletes than in controls in 9 studies and higher in athletes in 7 studies (figure 2). Only 3 of the 16 studies reported prevalence of hypertension in controls and 2 studies found more hypertension among athletes than controls. Overall, there was no significant difference in BP between athletes and controls.

Figure 3 shows the mean BP in different categories of athletes. Males had significantly higher BP than females (121.2 $\pm 4.5 / 75.1 \pm 2.9$ vs $113.5 \pm 2.9 / 71.9 \pm 2.6 \mathrm{~mm} \mathrm{Hg}, \mathrm{p}<0.05)$, but there was no significant difference in SBP between white and black athletes. We found that strength-trained athletes had higher BP than endurance-trained athletes $(131.3 \pm 5.3 / 77.3$ \pm 1.4 vs $118.6 \pm 2.8 / 71.8 \pm 1.2 \mathrm{~mm} \mathrm{Hg}, \mathrm{p}<0.05$ ), while there was a trend towards higher BP in athletes training $\geq 10 \mathrm{~h} /$ week

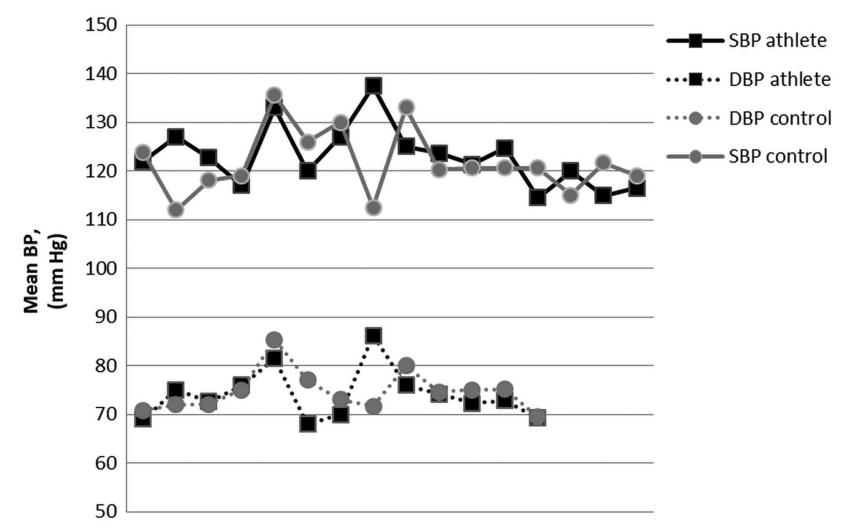

Figure 2 Mean systolic blood pressure (SBP; continuous line) and diastolic blood pressure (DBP; dotted line) in athletes (black squares) and controls (grey circles). compared with those training $<10 \mathrm{~h} /$ week $(121.8 \pm 3.8 / 73.8$ \pm 2.5 vs $117.6 \pm 3.3 / 66.8 \pm 6.9 \mathrm{~mm} \mathrm{Hg}, \mathrm{p}=0.058)$. There was no major difference between American football, soccer, triathlon and long distance running (figure 4).

\section{Prevalence of hypertension in athletes}

Hypertension was defined in 11 different ways in the 25 studies presenting a definition (table 2). The most often used criteria for hypertension ranged from SBP $\geq 140$ or DBP $\geq 90 \mathrm{~mm} \mathrm{Hg}$ to $\mathrm{BP}>140 / 90 \mathrm{~mm} \mathrm{Hg}$. The lowest cut-off value for hypertension was $\mathrm{BP} \geq 130 / 85 \mathrm{~mm} \mathrm{Hg}^{37}$ and the highest cut-off value was $\geq 160 / 95 \mathrm{~mm} \mathrm{Hg}^{27}$ Three studies also used antihypertensive medication to define hypertension, ${ }^{18} 2934$ one accepted selfreported hypertension ${ }^{18}$ and one only included participants with $\mathrm{BP} \leq 120 / 80 \mathrm{~mm} \mathrm{Hg}^{38}$

The prevalence of hypertension varied from $83 \%{ }^{34}$ to $0 \%$ (table 2). ${ }^{39}$ The prevalence of hypertension was lower in studies that were restricted to athletes within the age range 18-40 years and six studies excluded patients with high BP, mostly $>140 / 90 \mathrm{~mm} \mathrm{Hg} .{ }^{38-43}$

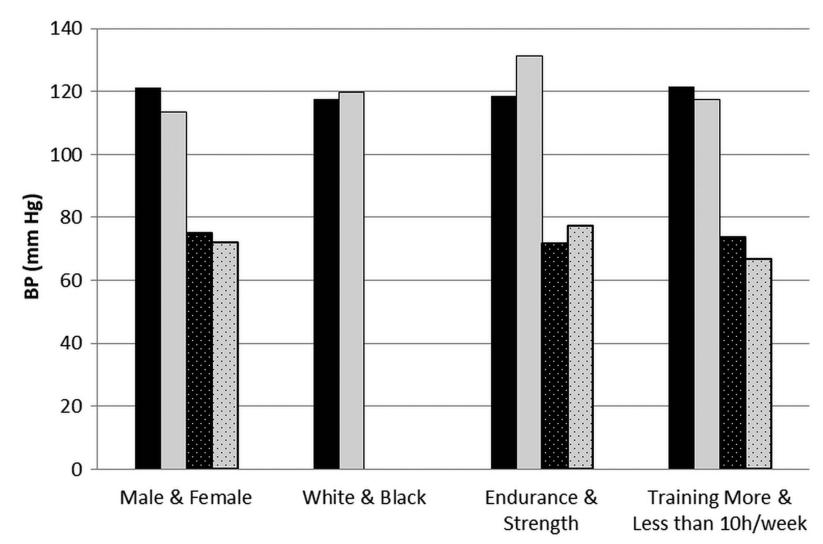

Figure 3 Blood pressure (BP) in relation to gender, ethnicity, type of training and hours of training per week. 


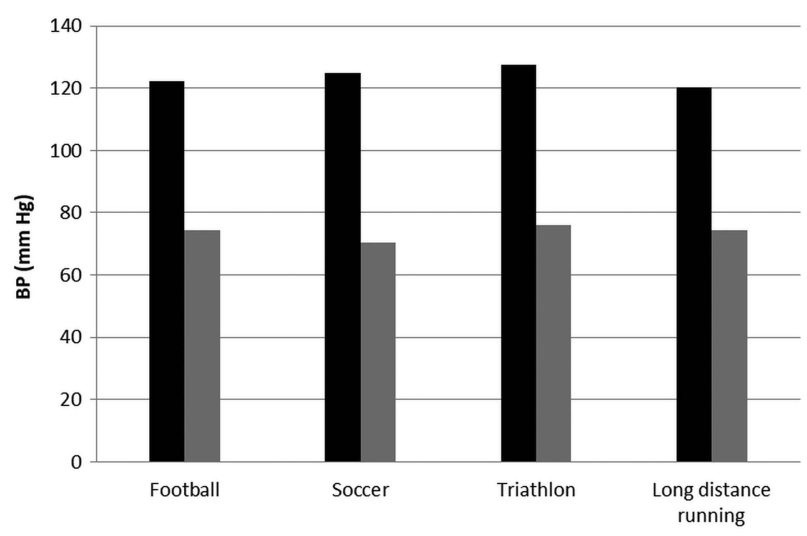

Figure 4 Blood pressure (BP) in different sports disciplines.

\section{Method of measurement of BP in athletes}

Some descriptions of measurement methods were present in 21 studies (figure 5; see online supplementary figure S3). BP was measured in the sitting position in 10 studies and in a supine position in 6 studies. At least $5 \mathrm{~min}$ of rest prior to BP recordings was required in 11 studies, while only 4 informed about time from physical activity to BP measurement. ${ }^{6} \quad 13 \quad 44 \quad 45$ Athletes abstained from caffeine and/or smoking prior to BP recordings in two studies ${ }^{64}$ and no studies informed about the physical environment where the BP measurements took place. Only eight studies reported whether an appropriate cuff size was used. In the eight studies using a 'standard' mercury sphygmomanometer, the method of measurement performance was reported in three studies. ${ }^{45-47}$ Only the three studies that used an automated BP device reported the device type and manufacturer. $^{132629}$ A single measurement was used in five studies, but repeated in three of these if BP was high. The lowest of these values was registered in two studies ${ }^{18} 48$ and the highest in one. ${ }^{27} \mathrm{BP}$ was recorded two and three times in six and four studies, respectively, and there was a significant difference in SBP between one and two BP recordings $(127 \pm 4.7$ vs $118 \pm 4.0$ $\mathrm{mm} \mathrm{Hg}, \mathrm{p}<0.05)$. Choice of arm for measurement was presented in five studies and no study measured BP in both arms. Three studies recommended repeated BP recordings on a separate occasion if the BP was elevated. Only one study referred athletes with elevated office BP to ambulatory BP measurement. ${ }^{49}$

\section{Association between BP and left ventricular hypertrophy}

Three of the four studies relating high BP to left ventricular hypertrophy showed a significant positive linear association, either between BP and indexed left ventricular mass, ${ }^{13}$ between resting SBP and left ventricle mass and left ventricle wall thickness, ${ }^{49}$ or between SBP and the RaVL lead in ECG. ${ }^{29}$ One study found no association between SBP and relative wall thickness. ${ }^{30}$

\section{DISCUSSION}

The most striking finding in this review was that the methods of BP measurement in athletes were poorly standardised and varied widely. The Seventh report of the Joint National Committee on Prevention, Detection, Evaluation, and Treatment of High Blood Pressure states that at least two measurements shall be made and the averaged recorded, ${ }^{33}$ and the European guidelines state that BP shall be measured three times after 5-10 min rest in the sitting position and the mean of the last two measurements shall be registered. ${ }^{21}$ The IOC has recommended BP recordings from both arms during preparticipation cardiac screening. ${ }^{50}$ All guidelines also recommend use of ambulatory BP measurements, but nearly all studies in our review were negligent to all these recommendations, as was a study among general practitioners in the UK. ${ }^{51}$

Given this background, it was difficult to give an estimate of BP or prevalence of hypertension in athletes. Naturally, the prevalence will be dependent on the definition of hypertension and varied from $0 \%$ to $83 \%$ in a subgroup of heavy weightlifters. The study with the lowest cut-off value $(\geq 130 / 85 \mathrm{~mm} \mathrm{Hg})$ also had the highest overall prevalence of hypertension $(45.1 \%$,

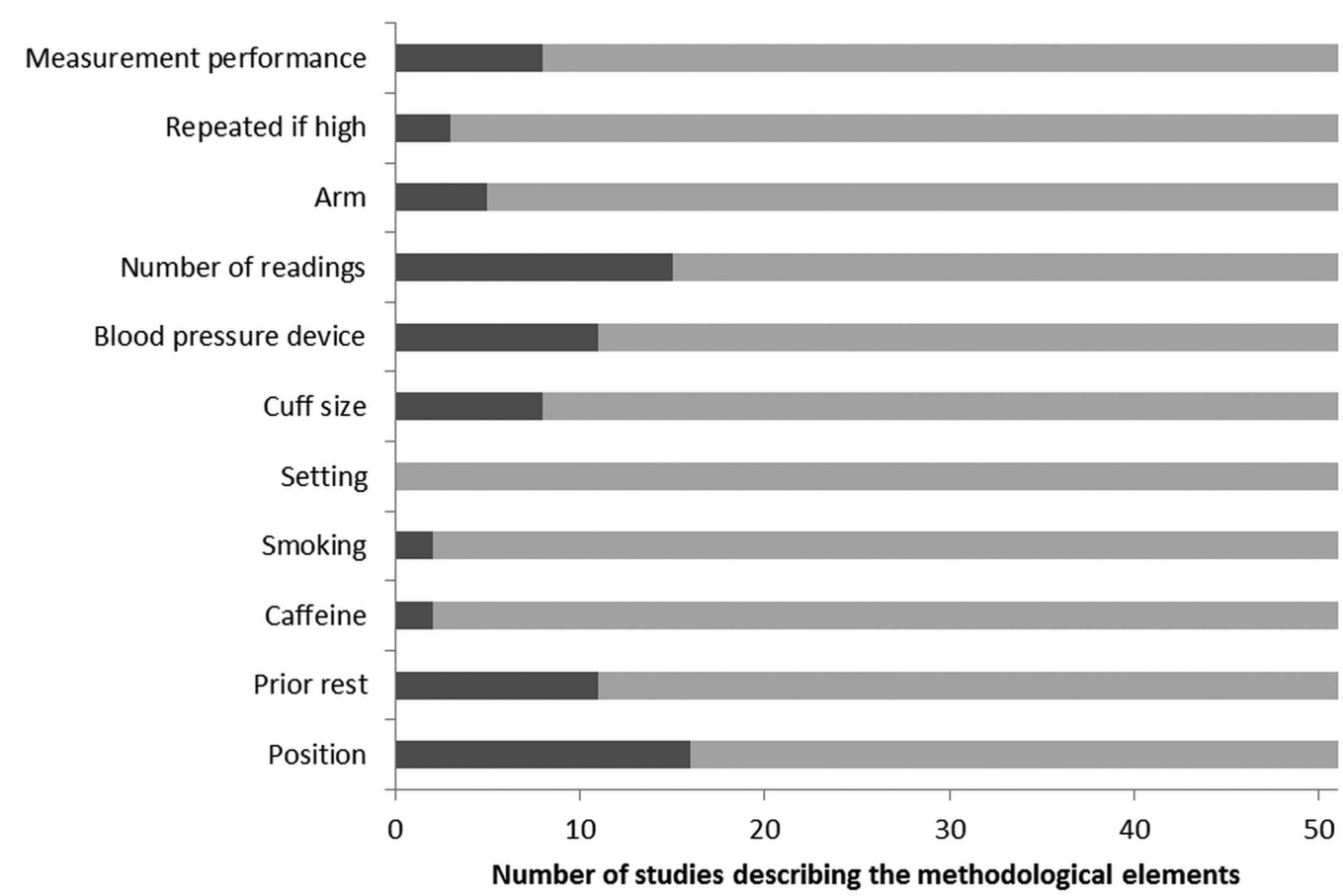

Figure 5 Number of studies describing each of the recommended elements in blood pressure measurements. 
compared with $17 \%$ in an age-matched and gender-matched control group). ${ }^{37}$ The study with the highest cut-off value for hypertension $(\geq 160 / 90 \mathrm{~mm} \mathrm{Hg})$ still reported a prevalence of $9.5 \%,{ }^{27}$ but this study included athletes with the highest age (36.4 \pm 4.5 years), and selectively reported the highest of the measured BPs.

Two-thirds of the studies reported mean BP in the prehypertensive range. There are several possible explanations for this. First, in many of these studies, BP was measured only once and one recording is often higher than the mean of two recordings. Second, the cuff size might have been too small for the muscular upper arms of athletes, which means that BP is measured falsely too high since only a minority of studies reported if they had used an appropriate cuff size. Third, the environment was probably not quiet in most studies and the athletes had no rest prior to the BP recordings. Fourth, the BP was recorded postseason. In a recent study of 132 professional American-style football participants, both SBP and DBP increased significantly from before to after the season. ${ }^{14}$ There is also the possibility that many athletes do have BP in the prehypertensive range, as suggested by several studies in this review and as supported by the trend towards a higher BP in athletes training $\geq 10 \mathrm{~h} /$ week than in those training $<10 \mathrm{~h} /$ week. Physical activity has a well-known BP-lowering effect in the general population ${ }^{33}$ and studies of 'exercise as medicine' report a decrease in BP of $4-9 \mathrm{~mm} \mathrm{Hg}^{33}$; but the amount of physical activity in these studies is often limited to $30 \mathrm{~min}$ most days a week. In our review, most athletes were 'elite' or 'professional', training on average $14.4 \mathrm{~h}$ per week or had been training vigorously for an average of 8.8 years, and it may well be that BP reacts differently to such amounts of training than to more moderate amounts of physical activity. There can be many biological reasons for high BP in athletes. First, the mental stress associated with competition at a high level might increase the athletes' BP, as indicated by the finding of a larger difference in SBP between professional athletes and controls $(4.4 \mathrm{~mm} \mathrm{Hg}, \mathrm{p}=0.350)$, than that between non-professional athletes and controls $(0.2 \mathrm{~mm} \mathrm{Hg}, \mathrm{p}=0.916$, data not shown). Second, BP might be increased due to 'spurious systolic hypertension' when BP is measured in the upper arm in athletes. ${ }^{52}$ Third, some athletes might use BP-increasing drugs, as shown in several studies, ${ }^{18}{ }^{53}$ which is an argument for collecting information about use of medication during preparticipation screening of athletes. ${ }^{33}$

We also found interesting differences between subgroups of athletes. For example, male athletes had significantly higher BP than female athletes; this was also found in a small study of 15 pairs in sports dancing, which showed that male dancers had significantly higher BP than their female counterparts, despite similar levels of training. ${ }^{54}$ We also found higher BP and a higher prevalence of hypertension in strength-trained athletes than in endurance-trained athletes, in accordance with the 'Morganroth hypothesis' ${ }^{55}$ The highest prevalence of hypertension, of $83.0 \%$, was found in professional male Chinese strength athletes, predominantly weightlifters, in the unlimited maximum body weight class. $^{33}$

There is increasing concern about the effects of vigorous, long-term athletic training on cardiovascular health ${ }^{56-58}$ and it is possible that some of the harmful effects may be mediated through high BP. High BP in adulthood increases risk of cardiovascular disease in the general population ${ }^{2}{ }^{3}$; we and others have found an association between high BP and left ventricular hypertrophy in athletes. ${ }^{13} 5459$ Whether this is a benign physiological adaptation to high BP or a beginning of pathological remodelling is not known. It may also be that left ventricular hypertrophy provokes hypertension or that other factors confound the association between high BP and left ventricular hypertrophy. High BP may also be a part of the explanation for the fivefold increased risk of atrial fibrillation in endurance athletes $^{15} \quad 60-62$ and exercise-induced arrhythmogenic right ventricular cardiomyopath ${ }^{56}$ through repeated bouts of high BP on myocyte junctions in the atria and the ventricles.

\section{Clinical impact and conclusions}

$\mathrm{BP}$ and prevalence of hypertension in athletes varies considerably partly because of variations in measurement methods, but type and intensity of training seem to play a role. Strength-trained athletes have significantly higher BP than endurance-trained athletes and vigorous physical activity does not seem to reduce BP in athletes compared with controls. Some studies found an association between high BP and left ventricular hypertrophy, but the clinical impact of high BP in athletes is not known. Future studies should adhere more rigorously to the recommendations for measurement of BP and should be designed to determine more precisely the prevalence, determinants and prognostic significance of hypertension in athletes.

Contributors All authors contributed to study design, discussion of the data and the final manuscript. CBI performed the systematic searches and extracted the data. HMB controlled the extracted data, performed the analyses and wrote the first draft of the manuscript.

Competing interests None.

Provenance and peer review Not commissioned; externally peer reviewed.

\section{REFERENCES}

1 Kearney PM, Whelton M, Reynolds K, et al. Global burden of hypertension: analysis of worldwide data. Lancet 2005;365:217-23.

2 Sundstrom J, Neovius M, Tynelius $\mathrm{P}$, et al. Association of blood pressure in late adolescence with subsequent mortality: cohort study of Swedish male conscripts. BMJ 2011;342:d643.

3 Gray L, Lee IM, Sesso HD, et al. Blood pressure in early adulthood, hypertension in middle age, and future cardiovascular disease mortality: HAHS (Harvard Alumni Health Study). J Am Coll Cardiol 2011;58:2396-403.

4 Sealy DP, Pekarek L, Russ D, et al. Vital signs and demographics in the preparticipation sports exam: do they help us find the elusive athlete at risk for sudden cardiac death? Curr Sports Med Rep 2010;9:338-41.

5 Lively MW. Preparticipation physical examinations: a collegiate experience. Clin J Sport Med 1999;9:3-8.

6 De Matos LD, Caldeira NA, Perlingeiro PS, et al. Cardiovascular risk and clinical factors in athletes: 10 years of evaluation. Med Sci Sports Exerc 2011;43:943-50.

7 Corrado D, Basso C, Schiavon M, et al. Pre-participation screening of young competitive athletes for prevention of sudden cardiac death. J Am Coll Cardiol 2008;52:1981-9.

8 Leddy JJ, Izzo J. Hypertension in athletes. J Clin Hypertens (Greenwich) 2009;11:226-33.

9 Pelliccia A, Fagard R, Bjornstad HH, et al. Recommendations for competitive sports participation in athletes with cardiovascular disease: a consensus document from the Study Group of Sports Cardiology of the Working Group of Cardiac Rehabilitation and Exercise Physiology and the Working Group of Myocardial and Pericardial Diseases of the European Society of Cardiology. Eur Heart $J$ 2005;26:1422-45.

10 Levy D, Garrison RJ, Savage DD, et al. Prognostic implications of echocardiographically determined left ventricular mass in the Framingham Heart Study. N Engl J Med 1990;322:1561-6.

11 Manolis AJ, Rosei EA, Coca A, et al. Hypertension and atrial fibrillation: diagnostic approach, prevention and treatment. Position paper of the Working Group 'Hypertension Arrhythmias and Thrombosis' of the European Society of Hypertension. J Hypertens 2012;30:239-52.

12 Maron BJ, Pelliccia A. The heart of trained athletes: cardiac remodeling and the risks of sports, including sudden death. Circulation 2006;114:1633-44.

13 Berge HM, Gjerdalen GF, Andersen TE, et al. Blood pressure in professional male football players in Norway. J Hypertens 2013;31:672-9.

14 Weiner RB, Wang F, Isaacs SK, et al. Blood pressure and left ventricular hypertrophy during American-style football participation. Circulation 2013;128:524-31.

15 Grimsmo J, Grundvold I, Maehlum S, et al. High prevalence of atrial fibrillation in long-term endurance cross-country skiers: echocardiographic findings and possible 
predictors - a 28-30 years follow-up study. Eur I Cardiovasc Prev Rehabil 2010;17:100-5.

16 Molina L, Mont L, Marrugat J, et al. Long-term endurance sport practice increases the incidence of lone atrial fibrillation in men: a follow-up study. Europace 2008; 10:618-23.

17 Milne C, Waddell R, Trease L, et al. Mythbusters in rowing medicine and physiotherapy: nine experts tackle five clinical conundrums. $\mathrm{Br} / \mathrm{Sports} \mathrm{Med}$ 2014:48:1525-8.

18 Karpinos AR, Roumie $\mathrm{CL}$, Nian $\mathrm{H}$, et al. High prevalence of hypertension among collegiate football athletes. Circ Cardiovasc Qual Outcomes 2013;6:716-23.

19 Berge HM, Andersen TE, Solberg EE, et al. High ambulatory blood pressure in male professional football players. Br J Sports Med 2013;47:521-5.

20 Ljungqvist A, Jenoure P, Engebretsen L, et al. The International Olympic Committee (IOC) Consensus Statement on periodic health evaluation of elite athletes March 2009. Br J Sports Med 2009:43:631-43.

21 Mancia G, De BG, Dominiczak A, et al. 2007 Guidelines for the Management of Arterial Hypertension: the task force for the management of arterial hypertension of the European Society of Hypertension (ESH) and of the European Society of Cardiology (ESC). J Hypertens 2007;25:1105-87.

22 Babaee Bigi MA, Aslani A. Aortic root size and prevalence of aortic regurgitation in elite strength trained athletes. Am J Cardiol 2007;100:528-30.

23 Corrado D, Basso C, Pavei A, et al. Trends in sudden cardiovascular death in young competitive athletes after implementation of a preparticipation screening program. JAMA 2006;296:1593-601.

24 Maskhulia L, Chabashvili N, Kakhabrishvili Z, et al. Electrocardiographic patterns and systolic and diastolic functions of the heart in the highly trained football players with increased left ventricular mass. Georgian Med News 2006;3:76-80.

25 Schmied C, Di Paolo FM, Zerguini AY, et al. Screening athletes for cardiovascular disease in Africa: a challenging experience. Br J Sports Med 2013;47:579-84.

26 Maldonado J, Pereira T, Polonia J, et al. Modulation of arterial stiffness with intensive competitive training. Rev Port Cardiol 2006;25:709-14.

27 Rontoyannis GP, Stalikas A, Sarros G, et al. Medical, morphological and functional aspects of Greek football referees. J Sports Med Phys Fitness 1998;38:208-14.

28 Thunenkotter T, Schmied C, Dvorak J, et al. Benefits and limitations of cardiovascular pre-competition screening in international football. Clin Res Cardiol 2010;99:29-35.

29 Tucker AM, Vogel RA, Lincoln AE, et al. Prevalence of cardiovascular disease risk factors among National Football League players. JAMA 2009;301:2111-19.

30 Douglas PS, O'Toole ML, Katz SE, et al. Left ventricular hypertrophy in athletes. Am J Cardiol 1997:80:1384-8.

31 Sharwood KA, Collins M, Goedecke JH, et al. Weight changes, medical complications, and performance during an Ironman triathlon. Br J Sports Med 2004;38:718-24.

32 Miranda-Vilela AL, Pereira LC, Goncalves CA, et al. Pequi fruit (Caryocar brasiliense Camb.) pulp oil reduces exercise-induced inflammatory markers and blood pressure of male and female runners. Nutr Res 2009:29:850-8.

33 Chobanian AV, Bakris GL, Black HR, et al. Seventh report of the Joint National Committee on Prevention, Detection, Evaluation, and Treatment of High Blood Pressure. Hypertension 2003;42:1206-52.

34 Guo J, Zhang X, Wang L, et al. Prevalence of metabolic syndrome and its components among Chinese professional athletes of strength sports with different body weight categories. PLOS ONE 2013;8:e79758.

35 D'Andrea A, Limongelli G, Caso P, et al. Association between left ventricular structure and cardiac performance during effort in two morphological forms of athlete's heart. Int I Cardiol 2002:86:177-84.

36 Noseworthy PA, Weiner R, Kim J, et al. Early repolarization pattern in competitive athletes: clinical correlates and the effects of exercise training. Circ Arrhythm Electrophysiol 2011:4:432-40.

37 Helzberg JH, Waeckerle JF, Camilo J, et al. Comparison of cardiovascular and metabolic risk factors in professional baseball players versus professional football players. Am J Cardiol 2010;106:664-7.

38 Zaidi A, Ghani S, Sharma R, et al. Physiologic right ventricular adaptation in elite athletes of African and Afro-Caribbean origin. Circulation 2013:127:1783-92.

39 Zaidi A, Ghani S, Sheikh N, et al. Clinical significance of electrocardiographic right ventricular hypertrophy in athletes: comparison with arrhythmogenic right ventricular cardiomyopathy and pulmonary hypertension. Eur Heart J 2013;34:3649-56.

40 Pelliccia A, Kinoshita N, Pisicchio C, et al. Long-term clinical consequences of intense, uninterrupted endurance training in Olympic athletes. J Am Coll Cardiol 2010;55:1619-25.

41 Papadakis M, Carre F, Kervio G, et al. The prevalence, distribution, and clinical outcomes of electrocardiographic repolarization patterns in male athletes of African/ Afro-Caribbean origin. Eur Heart J 2011;32:2304-13.

42 Caselli S, Di PR, Di Paolo FM, et al. Left ventricular systolic performance is improved in elite athletes. Eur J Echocardiogr 2011:12:514-19.

43 Gati S, Sheikh N, Ghani S, et al. Should axis deviation or atrial enlargement be categorised as abnormal in young athletes? The athlete's electrocardiogram: time for re-appraisal of markers of pathology. Eur Heart J 2013;34:3641-8.
44 Siegel D, Benowitz N, Ernster VL, et al. Smokeless tobacco, cardiovascular risk factors, and nicotine and cotinine levels in professional baseball players. Am $J$ Public Health 1992;82:417-21.

45 Varga-Pinter B, Horvath $\mathrm{P}$, Kneffel $Z$, et al. Resting blood pressure values of adult athletes. Kidney Blood Press Res 2011;34:387-95.

46 Berry WT, Beveridge JB, . The diet, haemoglobin values, and blood pressure of Olympic athletes. Br Med J 1949;1:300-4.

47 D'Andrea A, Cocchia R, Riegler $L$, et al. Aortic stiffness and distensibility in top-level athletes. J Am Soc Echocardiogr 2012;25:561-7.

48 Andersen $\mathrm{KL}$, Elvik A. The resting arterial blood pressure in athletes. Acta Med Scand 1956;153:367-71

49 Riding NR, Salah O, Sharma S, et al. ECG and morphologic adaptations in Arabic athletes: are the European Society of Cardiology's recommendations for the interpretation of the 12-lead ECG appropriate for this ethnicity? Br I Sports Med 2014;48:1138-43.

50 Ljungqvist A, Jenoure PJ, Engebretsen L, et al. The International Olympic Committee (IOC) consensus statement on periodic health evaluation of elite athletes. Clin $J$ Sport Med 2009;19:347-65.

51 Heneghan $C$, Perera R, Mant $D$, et al. Hypertension quideline recommendations in general practice: awareness, agreement, adoption, and adherence. $\mathrm{Br} / \mathrm{Gen}$ Pract 2007:57:948-52

52 Palatini P. Cardiovascular effects of exercise in young hypertensives. Int I Sports Med 2012;33:683-90

53 Malhotra R, West JJ, Dent J, et al. Cost and yield of adding electrocardiography to history and physical in screening Division I intercollegiate athletes: A 5-year experience. Heart Rhythm 2011;8:721-7.

54 Zemva A, Rogel P. Gender differences in athlete's heart: association with 24-h blood pressure. A study of pairs in sport dancing. Int I Cardiol 2001;77:49-54

55 Naylor LH, George K, O'Driscoll G, et al. The athlete's heart: a contemporary appraisal of the 'Morganroth hypothesis'. Sports Med 2008;38:69-90.

56 Heidbuchel H, Prior DL, La GA. Ventricular arrhythmias associated with long-term endurance sports: what is the evidence? Br J Sports Med 2012;46(Suppl 1):i44-50.

57 O'Keefe JH, Patil HR, Lavie CJ, et al. Potential adverse cardiovascular effects from excessive endurance exercise. Mayo Clin Proc 2012;87:587-95.

58 Elliott $A D$, La GA. The right ventricle following prolonged endurance exercise: are we overlooking the more important side of the heart? A meta-analysis. $\mathrm{Br} /$ Sports Med 2014 Oct 3. doi:10.1136/bjsports-2014-093895. [Epub ahead of print].

59 Wilson MG, Chatard JC, Carre F, et al. Prevalence of electrocardiographic abnormalities in West-Asian and African male athletes. Br I Sports Med 2012:46:341-7

60 Abdulla J, Nielsen JR. Is the risk of atrial fibrillation higher in athletes than in the general population? A systematic review and meta-analysis. Europace 2009;11:1156-9.

61 Pelliccia A, Maron BJ, Di Paolo FM, et al. Prevalence and clinical significance of left atrial remodeling in competitive athletes. I Am Coll Cardiol 2005:46:690-6.

62 Eshoo S, Ross DL, Thomas L. Impact of mild hypertension on left atrial size and function. Circ Cardiovasc Imaging 2009;2:93-9.

63 van Buuren F, Mellwig KP, Butz T, et al. Left ventricular mass and oxygen uptake in top handball athletes. Int I Sports Med 2013;34:200-6

64 Sofi F, Capalbo A, Pucci N, et al. Cardiovascular evaluation, including resting and exercise electrocardiography, before participation in competitive sports: cross sectional study. BMJ 2008:337:a346.

65 Munoz L, Norgan G, Rauschhuber M, et al. An exploratory study of cardiac health in college athletes. Appl Nurs Res 2009;22:228-35.

66 Lewis JF, Maron BJ, Diggs JA, et al. Preparticipation echocardiographic screening for cardiovascular disease in a large, predominantly black population of collegiate athletes. Am J Cardiol 1989;64:1029-33.

67 Di Luigi L, Pelliccia A, Bonetti A, et al. Clinical efficacy and preventive role of the pre-participation physical examination in Italy. Med Sport 2004;57:243-70.

68 Magalski A, McCoy M, Zabel M, et al. Cardiovascular screening with electrocardiography and echocardiography in collegiate athletes. Am J Med 2011;124:511-18.

69 Maron BJ, Bodison SA, Wesley YE, et al. Results of screening a large group of intercollegiate competitive athletes for cardiovascular disease. J Am Coll Cardiol 1987:10:1214-21.

70 Urhausen A, Monz T, Kindermann W. Sports-specific adaptation of left ventricular muscle mass in athlete's heart. I. An echocardiographic study with combined isometric and dynamic exercise trained athletes (male and female rowers). Int J Sports Med 1996;17(Suppl 3):S145-51.

71 Pelliccia A, Maron BJ, Culasso F, et al. Clinical significance of abnormal electrocardiographic patterns in trained athletes. Circulation 2000;102:278-84.

72 Pougnet R, Costanzo LD, Lodde B, et al. Cardiovascular risk factors and cardiovascular risk assessment in professional divers. Int Marit Health 2012;63:164-9 


\section{Review}

73 Schmied C, Notz S, Cribari M, et al. Cardiac pre-competition screening in Swiss athletes. Current situation in competitive athletes and short-time assessment of an exemplary local screening program. Swiss Med Wkly 2012;142:w13575.

74 Abergel E, Chatellier G, Hagege AA, et al. Serial left ventricular adaptations in world-class professional cyclists: implications for disease screening and follow-up. J Am Coll Cardiol 2004;44:144-9.

75 Basavarajaiah S, Boraita A, Whyte $G$, et al. Ethnic differences in left ventricular remodeling in highly-trained athletes relevance to differentiating physiologic left ventricular hypertrophy from hypertrophic cardiomyopathy. I Am Coll Cardiol 2008:51:2256-62.
76 Pagourelias ED, Kouidi E, Efthimiadis GK, et al. Right atrial and ventricular adaptations to training in male Caucasian athletes: an echocardiographic study. J Am Soc Echocardiogr 2013;26:1344-52.

77 Vitarelli A, Capotosto L, Placanica G, et al. Comprehensive assessment of biventricular function and aortic stiffness in athletes with different forms of training by three-dimensional echocardiography and strain imaging. Eur Heart J Cardiovasc Imaging 2013;14:1010-20.

78 Chandra N, Bastiaenen R, Papadakis $M$, et al. The prevalence of ECG anomalies in young individuals; relevance to a nationwide cardiac screening program. J Am Coll Cardiol 2014;63:2028-34. 